Research Article

\title{
Determination of Position Resolution for LYSO Scintillation Crystals Using Geant4 Monte Carlo Code
}

\author{
M. F. O. Yahya and F. Kocak (iD \\ Department of Physics, Bursa Uludag University, 16059 Bursa, Turkey \\ Correspondence should be addressed to F. Kocak; fkocak@uludag.edu.tr
}

Received 1 April 2021; Revised 14 July 2021; Accepted 26 August 2021; Published 15 September 2021

Academic Editor: Burak Bilki

Copyright (c) 2021 M. F. O. Yahya and F. Kocak. This is an open access article distributed under the Creative Commons Attribution License, which permits unrestricted use, distribution, and reproduction in any medium, provided the original work is properly cited. The publication of this article was funded by SCOAP ${ }^{3}$.

LYSO scintillation crystals, due to their significant characteristics such as high light yield, fast decay time, small Moliére radius, and good radiation hardness, are proposed to be used for the electromagnetic calorimeter section of the Turkish Accelerator Center Particle Factory (TAC-PF) detector. In this work, the center of gravity technique was used to determine the impact coordinates of an electron initiating an electromagnetic shower in a LYSO array, in a calorimeter module containing nine crystals, each $25 \mathrm{~mm} \times 25 \mathrm{~mm}$ in cross-section and $200 \mathrm{~mm}$ in length. The response of the calorimeter module has been studied with electrons having energies in the range $0.1 \mathrm{GeV}-2 \mathrm{GeV}$. By using the Monte Carlo simulation based on Geant4, the twodimensional position resolution of the module is obtained as $\sigma_{R}(\mathrm{~mm})=((3.95 \pm 0.08) / \sqrt{E}) \oplus(1.91 \pm 0.11)$ at the center of the crystal.

\section{Introduction}

Cerium-doped silicate-based heavy scintillation crystals were initially developed for medical applications. Later, the scintillation characteristics of lutetium oxyorthosilicate (LSO) [1] and lutetium-yttrium oxyorthosilicate (LYSO) $[2,3]$ were found. LYSO crystals have high stopping power $\left(>7 \mathrm{~g} / \mathrm{cm}^{3}\right)$, fast decay time $(40 \mathrm{~ns})$ and high light yield (200 times of PWO), and superior radiation hardness against gamma rays, neutrons, and protons [4-6]. Furthermore, the crystal emits light in the wavelength region between $360 \mathrm{~nm}$ and $600 \mathrm{~nm}$, reaching a peak at $402 \mathrm{~nm}$. Due to all of the above-mentioned properties, this crystal has also attracted the attention of experimental high energy physics research groups working to improve the performance of electromagnetic calorimeters, such as the proposed SuperB forward parts of the endcap calorimeter [7], the KLOE experiment [8], the COMET experiment at J-PARC [9], and the Muon-to-Electron (Mu2e) experiment [10]. In addition to lead tungstate (PWO) and Thallium-activated Cesium Iodide (CsI(Tl)) crystals, LYSO scintillation crystals may also be studied for the electromagnetic calorimeter (ECAL) section of the proposed Turkish Accelerator
Center-Particle Factory (TAC-PF) detector [11]. This paper reports the results of a simulation study carried out with Geant4 to evaluate the position resolution of LYSO scintillation crystals for incident electrons at an energy range from $0.1 \mathrm{GeV}$ to $2 \mathrm{GeV}$.

\section{Position Resolution of Electromagnetic Calorimeters}

When a particle is sent to the electromagnetic calorimeter, its energy is deposited in the central crystal as well as the crystals around it. The impact position of the particle can be found from the weighted mean of the position of energy deposits in the crystals. The technique used to find the position of the incident particle is called the center of gravity method and calculated by

$$
x_{\text {gravity }}=\frac{\sum_{i} x_{i} E_{i}}{\sum_{i} E_{i}}
$$

for the $x$-coordinate and similarly, 


$$
y_{\text {gravity }}=\frac{\sum_{i} y_{i} E_{i}}{\sum_{i} E_{i}},
$$

for the $y$-coordinate, where $E_{i}$ is the energy deposited in the $i^{\text {th }}$ crystal and $x_{i}$ and $y_{i}$ are the coordinates of the $i^{\text {th }}$ crystal [12]. For the ideal position resolution, it is preferable to take the sum over nine crystals due to the effect of large energy fluctuations in the tails of an electromagnetic cascade [13].

\section{Geant4 Simulation and Results}

Geant4 simulation code [14] was employed to perform the simulation process for the electrons passing the electromagnetic calorimeter module consisting of $3 \times 3$ LYSO scintillation crystals. The electrons were directed perpendicular to the module in the energy range of $0.1 \mathrm{GeV}$ to $2 \mathrm{GeV}$. The simulation was performed with Geant4.10.04-patch-03 with the QGSP-BERT4.5 physics list. The LYSO crystals have a length of $200 \mathrm{~mm}\left(17.5 X_{0}\right)$ with a cross-section of $25 \times 25$ $\mathrm{mm}^{2}\left(1.2 R_{M}\right)$. In the simulation, in order to obtain the distributions of the center of gravity of the deposited energies in the crystals, electrons were injected into the central crystal at fourteen different positions $(-12.5 \mathrm{~mm},-11 \mathrm{~mm},-9 \mathrm{~mm}$, $-7 \mathrm{~mm},-5 \mathrm{~mm},-3 \mathrm{~mm},-1 \mathrm{~mm}, 0,2 \mathrm{~mm}, 4 \mathrm{~mm}, 6 \mathrm{~mm}, 8$ $\mathrm{mm}, 10 \mathrm{~mm}$, and $12.5 \mathrm{~mm}$ ), respectively, to scan the entire surface of the crystal. The coordinates of the center of the central crystal were defined to be $x=0, y=0$. The relation between $x_{\text {gravity }}$ and $y_{\text {gravity }}$ in the center of the LYSO matrix for $1 \mathrm{GeV}$ electrons can be seen from Figure 1 .

The calculated positions $\left(x_{\text {gravity }}\right)$ versus the true positions $\left(x_{\text {true }}\right)$ are shown in Figure 2 for $1 \mathrm{GeV}$ electrons (Scurve). If the impact point is at the center of the crystal or near the boundary between different crystals, the particle's position can be reconstructed correctly, as can be seen from Figure 2. In other cases, since most of the energy in the shower is deposited in the hit crystal and there is an exponential fall in the energy shared among neighboring crystals, the position of the particle is systematically miscalculated from equation (1).

To remove this nonlinear dependence among the true positions $x_{\text {true }}$ and the calculated positions $x_{\text {gravity, }}$ the Scurve fit function was utilized. This function is an empirical algorithm and given by

$$
x_{\text {gravity }}=c \tan d\left(x_{\text {true }}-e\right)
$$

Here, $x_{\text {gravity }}$ and $x_{\text {true }}$ are presented in $\mathrm{mm}$. As a result of this fit, the values of $c, d$, and $e$ at $1 \mathrm{GeV}$ are found to be $2.669,0.108$, and 0.001 , respectively. Table 1 shows the obtained fit results for the incident electron energies between 0.1 and $2 \mathrm{GeV}$ in the $X$ direction. As the Moliére radius is loosely dependent on energy, the parameters differ slightly depending on the energy of the incident electron. By employing the values $c, d$, and $e$ which are obtained from

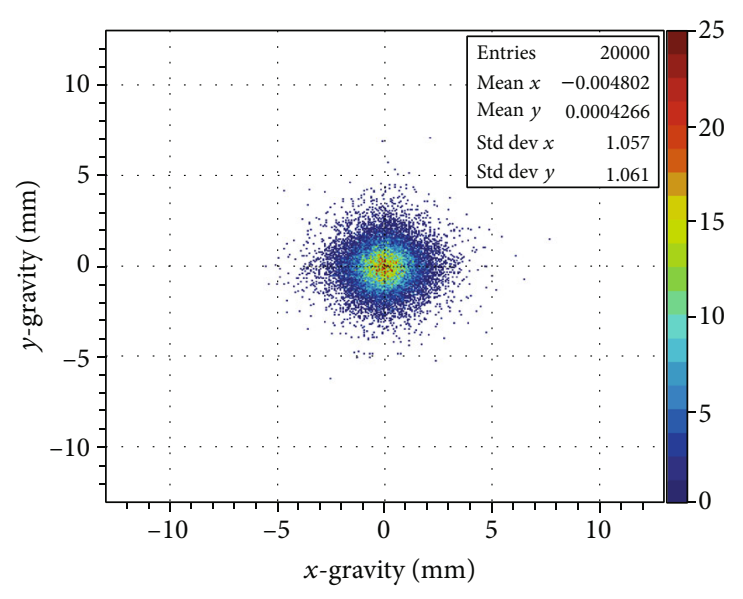

FIGURE 1: Relation between $x_{\text {gravity }}$ and $y_{\text {gravity }}$ in the center of the LYSO matrix for $1 \mathrm{GeV}$ electrons.

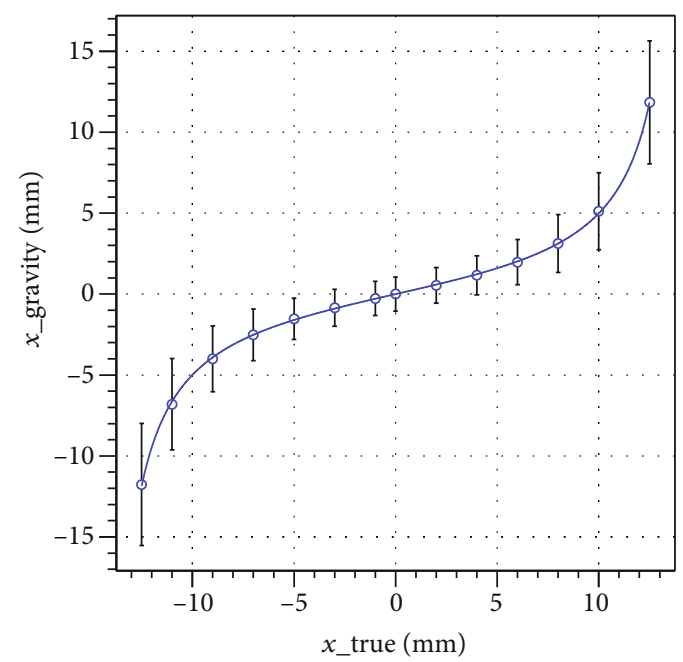

FIgURE 2: The $x_{\text {gravity }}$ position versus the $x_{\text {true }}$ position for $1 \mathrm{GeV}$ electrons. The solid blue line is the S-like curve.

the fit, the corrected $X$ position $\left(x_{\text {corrected }}\right)$ is calculated by

$$
x_{\text {corrected }}=\frac{1}{d} \tan ^{-1} \frac{x_{\text {gravity }}}{c}+e .
$$

Similar calculations have been also made for the $Y$ direction to obtain the corrected $Y$ position $\left(y_{\text {corrected }}\right)$. As the corrected position distribution spectra have roughly a Gaussian shape (see Figure 3), they have been fitted using a Gaussian function to obtain the position resolution. The sigmas of these distributions shown in Figure 3 give the calorimeter position resolutions in the $X$ and $Y$ directions for $1 \mathrm{GeV}$ electron energy. Figure 4 shows the corrected position $\left(x_{\text {corrected }}\right)$ versus the true position $\left(x_{\text {true }}\right)$ for $1 \mathrm{GeV}$ electrons in the $X$ direction. Figure 5 displays the position resolutions at the center of the central LYSO crystal (at coordinate $x=$ $y=0$ ) for the incident electron energies from $0.1 \mathrm{GeV}$ to $2 \mathrm{GeV}$. The Geant 4 simulations indicate that the position resolution improves as the energy of the incident electron 
TABle 1: Fit parameters for the incident electrons in the $X$ direction.

\begin{tabular}{lccc}
\hline Electron energy $(\mathrm{GeV})$ & $c(\mathrm{~mm})$ & $d(\mathrm{rad} / \mathrm{mm})$ & $e(\mathrm{~mm})$ \\
\hline 0.1 & 2.166 & 0.112 & -0.007 \\
0.25 & 2.327 & 0.110 & 0.017 \\
0.5 & 2.739 & 0.107 & -0.003 \\
0.75 & 2.702 & 0.108 & -0.004 \\
1 & 2.669 & 0.108 & 0.001 \\
1.25 & 2.637 & 0.108 & -0.023 \\
1.5 & 2.651 & 0.108 & -0.003 \\
1.75 & 2.654 & 0.108 & 0.003 \\
2 & 2.613 & 0.108 & -0.01 \\
\hline
\end{tabular}
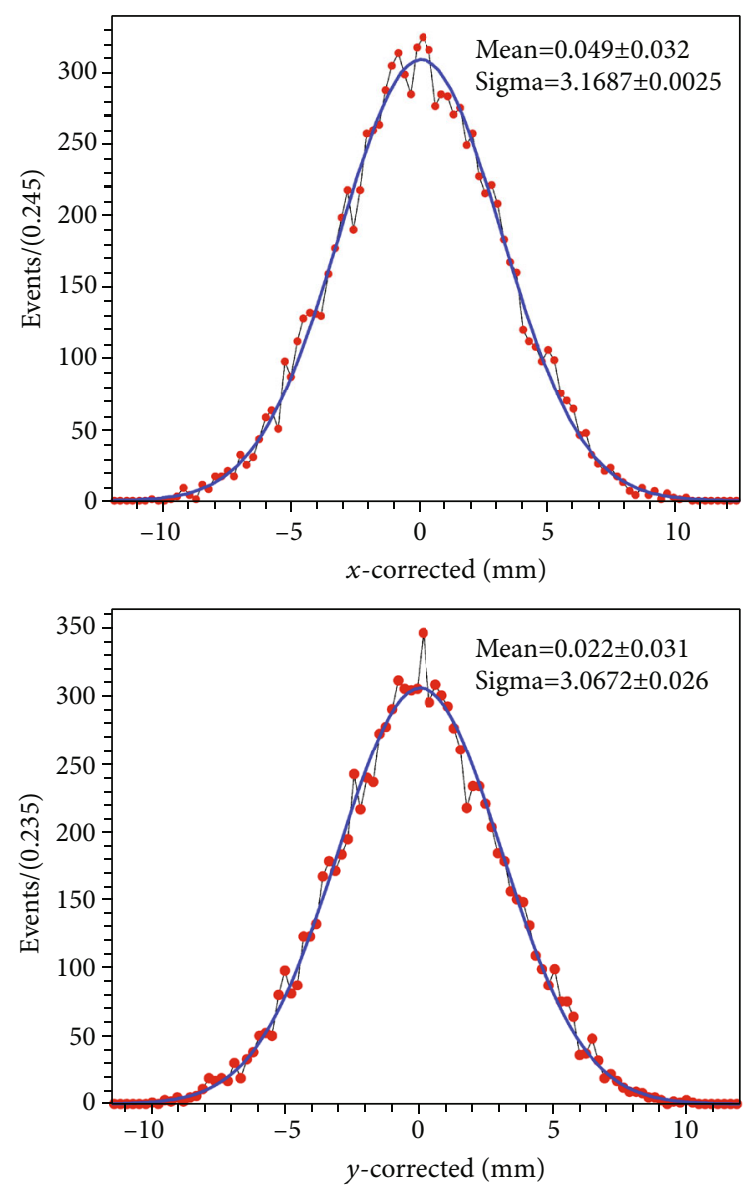

Figure 3: $x_{\text {corrected }}$ and $y_{\text {corrected }}$ positions after the S-curve correction, for electron energy of $1 \mathrm{GeV}$.

increases as shown in Figure 5. At the center of the central crystal, the position resolutions depending on the energy of the incident electrons can be parameterized as

$$
\sigma_{x}(\mathrm{~mm})=\frac{(2.77 \pm 0.07)}{\sqrt{E}} \oplus(1.46 \pm 0.10)
$$

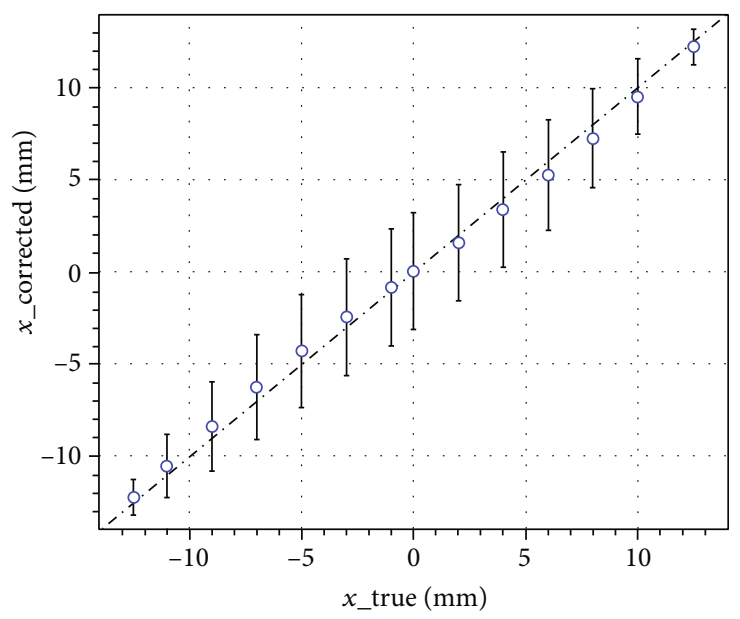

FIGURE 4: Dependence of the corrected position $\left(x_{\text {corrected }}\right)$ in the LYSO crystals on the true coordinate $\left(x_{\text {true }}\right)$.
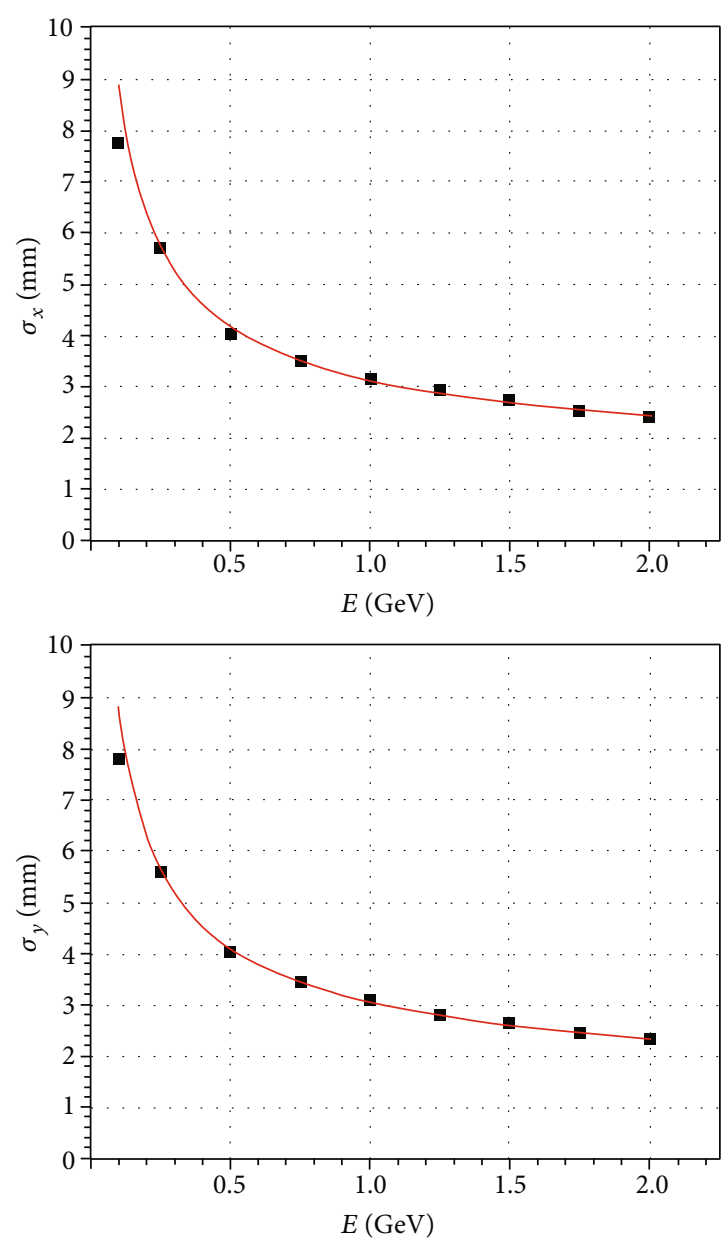

Figure 5: Position resolutions in the $X$ and $Y$ directions at the center of the $3 \times 3$ LYSO matrix as a function of the energy. The solid lines represent the fits to the data. The error bars are negligible compared to the symbols shown. 

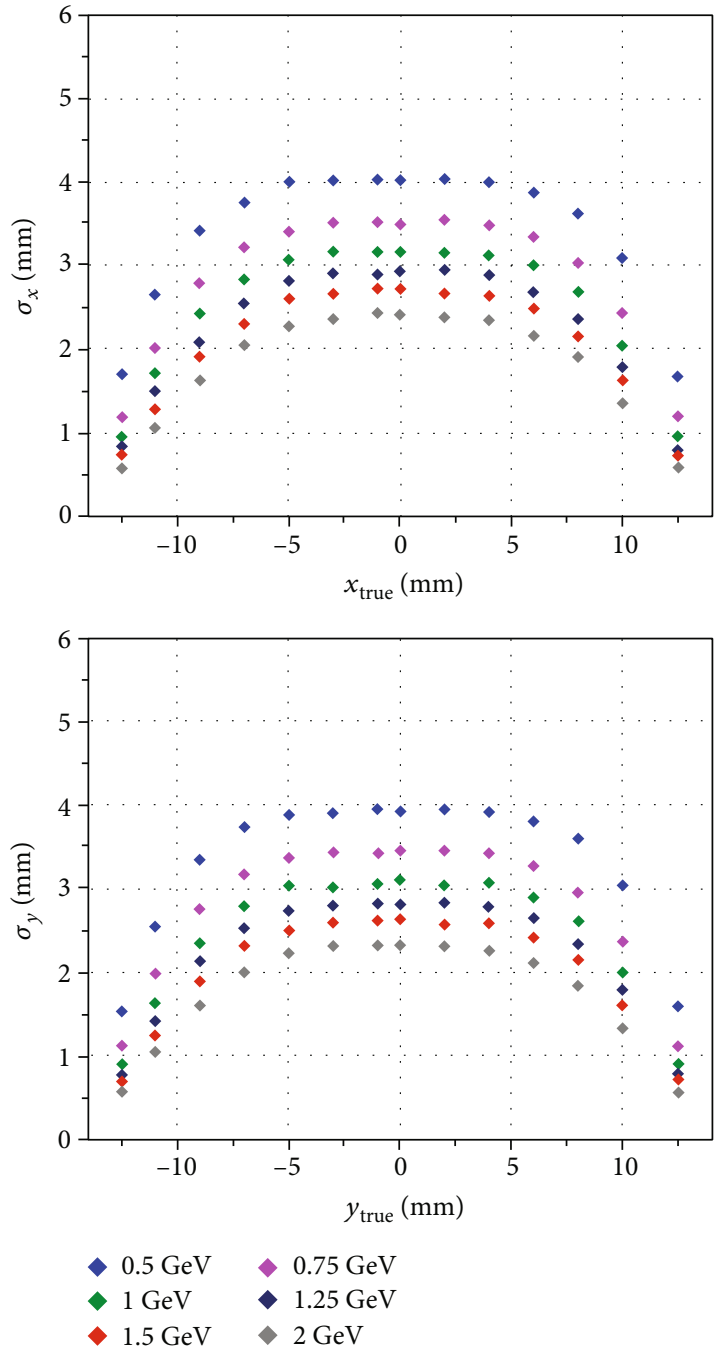

FIgURe 6: Position resolutions versus the impact position of the electron in the $X$ and $Y$ directions.

$$
\sigma_{y}(\mathrm{~mm})=\frac{(2.77 \pm 0.05)}{\sqrt{E}} \oplus(1.31 \pm 0.07)
$$

for the $Y$ direction.

The spatial resolution is not constant on the entire surface of the scintillation crystal and changes depending on the impact position of the incident electron. As can be seen in Figure 6, the simulated position resolution improves towards the edges of the crystal scintillator, and the minimum spatial resolutions are acquired on the edges. This is because the electromagnetic cascade sharing among neighboring crystals starts to become important in that position of the crystal.

One can get the two-dimensional position resolution $\sigma_{R}$ by using $\sigma_{x}$ and $\sigma_{y}$ values:

$$
\sigma_{R}(\mathrm{~mm})=\sqrt{\sigma_{x}^{2}+\sigma_{y}^{2}}
$$

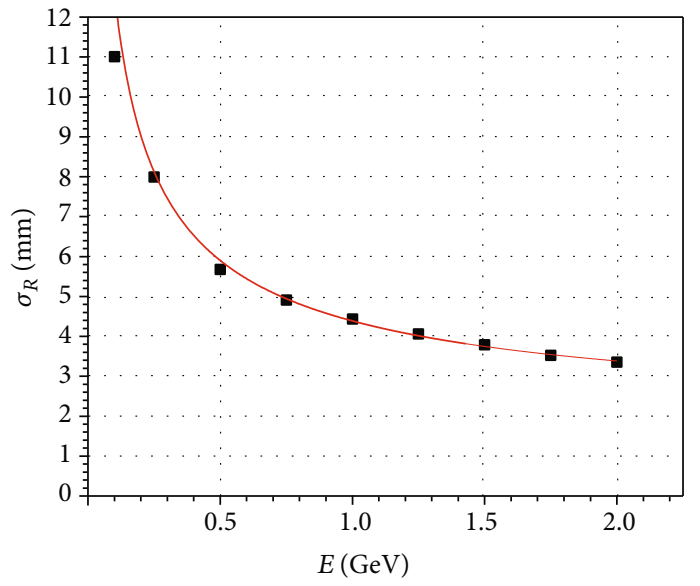

FIgURE 7: Dependence of the two-dimensional position resolution on electron energy.

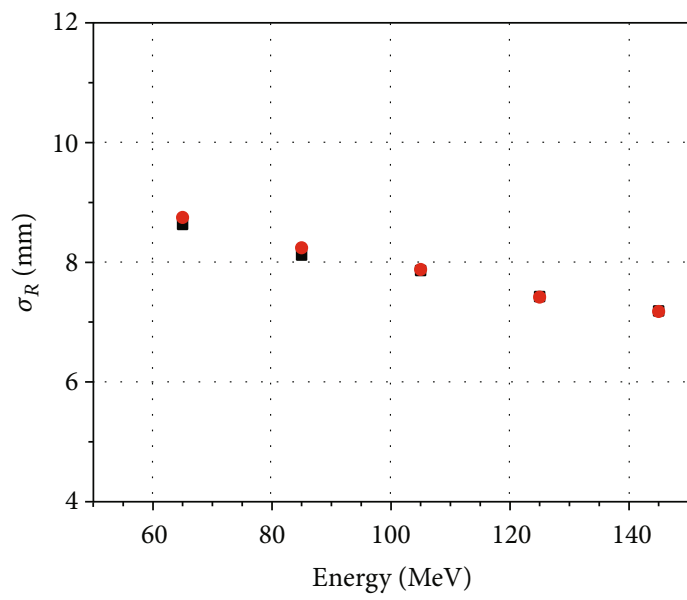

Geant4

K. Oishi [9]

Figure 8: Comparison of the position resolutions obtained from Ref. [9] with Geant4 simulation for the $7 \times 7$ crystal matrix.

Figure 7 shows the two-dimensional position resolution as a function of energy at the center of the $3 \times 3$ LYSO matrix.

In addition, in order to check the accuracy of the simulation code, a prototype electromagnetic calorimeter developed for the COMET experiment given in Ref. [9] was simulated with the Geant 4 code. The mentioned prototype consists of $7 \times 7$ LYSO crystals with a dimension of $2 \times 2 \times$ $12 \mathrm{~cm}^{3}$. The position resolution of the prototype was calculated for the incident electrons using the center of gravity technique mentioned above. The simulation results are well-matched with the experimental data with a slight difference at low energies as can be shown in Figure 8.

\section{Conclusion}

The simulation of the position resolution of the electromagnetic calorimeter module made of LYSO crystals for the 
proposed TAC-PF detector has been performed using Geant 4 simulation code. The position resolution was calculated every $2 \mathrm{~mm}$ step for the incident electron energies from 0.1 to $2 \mathrm{GeV}$, as can be shown in Figure 6. The resolution has improved significantly with S-shape correction. For electrons entering the calorimeter module in the center of the LYSO crystal, the resolution in the $X(Y)$ direction is $7.76 \mathrm{~mm}(7.81 \mathrm{~mm})$ at $0.1 \mathrm{GeV}$ and $2.41 \mathrm{~mm}(2.32 \mathrm{~mm})$ at $2 \mathrm{GeV}$. The position resolution was calculated as $\sigma_{x}(\mathrm{~mm})$ $=((2.77 \pm 0.07) / \sqrt{E}) \oplus(1.46 \pm 0.10)$ for the $X$ direction, and a similar result was also obtained for the $Y$ direction. The two-dimensional position resolution was calculated as $\sigma_{R}(\mathrm{~mm})=((3.95 \pm 0.08) / \sqrt{E}) \oplus(1.91 \pm 0.11)$. Also, by using Function (4) with the fit parameters shown in Table 1 with a minor modification, the impact position of an electron or photon in similar LYSO calorimeters can be deduced from the center of gravity of energy deposits in the crystals.

\section{Data Availability}

All figures in the paper represent the results obtained by Monte Carlo simulation, and the data used to support the findings of this study are cited at relevant places within the text as references.

\section{Conflicts of Interest}

The authors declare that they have no conflicts of interest.

\section{Acknowledgments}

The numerical calculations reported in this paper were partially performed at TUBITAK ULAKBIM, High Performance and Grid Computing Center (TRUBA resources).

\section{References}

[1] C. Melcher and J. Schweitzer, "Cerium-doped lutetium oxyorthosilicate: a fast, efficient new scintillator," IEEE Transactions on Nuclear Science, vol. 39, no. 4, pp. 502-505, 1992.

[2] D. Cooke, K. McClellan, B. Bennett et al., "Crystal growth and optical characterization of cerium-doped $\mathrm{Lu}_{1.8} Y_{0.2} \mathrm{SiO}_{5}$," Journal of Applied Physics, vol. 88, no. 12, pp. 7360-7362, 2000.

[3] T. Kimble, M. Chou, and B. H. T. Chai, "Scintillation properties of LYSO crystals," in 2002 IEEE Nuclear Science Symposium Conference Record, pp. 1434-1437, Norfolk, VA, USA, November 2002.

[4] R. Mao, L. Zhang, and R. Y. Zhu, "Gamma ray induced radiation damage in PWO and LSO/LYSO crystals," in 2009 IEEE Nuclear Science Symposium Conference Record (NSS/MIC), pp. 2045-2049, Orlando, FL, USA, October 2009.

[5] L. Zhang, R. Mao, and R. Y. Zhu, "Effects of neutron irradiations in various crystal samples of large size for future crystal calorimeter," in 2009 IEEE Nuclear Science Symposium Conference Record (NSS/MIC), pp. 2041-2044, Orlando, FL, USA, October 2009.
[6] G. Dissertori, D. Luckey, F. Nessi-Tedaldi et al., "Results on damage induced by high-energy protons in LYSO calorimeter crystals," Nuclear Instruments and Methods in Physics Research Section A, vol. 745, pp. 1-6, 2014.

[7] G. Eigen, Z. Zhou, D. Chao et al., "A LYSO calorimeter for the SuperB factory," Nuclear Instruments and Methods in Physics Research Section A, vol. 718, pp. 107-109, 2013.

[8] M. Cordelli, F. Happacher, M. Martini et al., "CCALT: a crystal calorimeter for the KLOE-2 experiment," Journal of Physics: Conference Series, vol. 293, no. 1, article 012010, 2011.

[9] K. Oishi, "Development of electromagnetic calorimeter using GSO and LYSO crystals for the J-PARC muon-to-electron conversion search experiment," in 2014 IEEE Nuclear Science Symposium and Medical Imaging Conference (NSS/MIC), pp. 1-6, Seattle, WA, USA, November 2014.

[10] R. Tschirhart, "The Mu2e experiment at Fermilab," Nuclear Physics B-Proceedings Supplements, vol. 210-211, pp. 245248, 2011.

[11] F. Kocak and I. Tapan, "Simulation of LYSO crystal for the TAC-PF electromagnetic calorimeter," Acta Physica Polonica A, vol. 131, no. 3, pp. 527-529, 2017.

[12] R. Wigmans, Calorimetry: Energy Measurement in Particle Physics, Oxford University Press, 2000.

[13] V. A. Batarin, T. Brennan, J. Butler et al., "Precision measurement of energy and position resolutions of the BTeV electromagnetic calorimeter prototype," Nuclear Instruments and Methods in Physics Research Section A: Accelerators, Spectrometers, Detectors and Associated Equipment, vol. 510, no. 3, pp. 248-261, 2003.

[14] S. Agostinelli, J. Allison, K. Amako et al., "Geant4-a simulation toolkit," Nuclear Instruments and Methods in Physics Research Section A: Accelerators, Spectrometers, Detectors and Associated Equipment, vol. 506, no. 3, pp. 250-303, 2003. 